\title{
TRANSLATION SERVICES: A BRIEF STUDY
}

\author{
Subhajit Panda \\ Student, B.Lib.I.Sc., Panjab University, Chandigarh \\ Email: suvapanda007@gmail.com
}

\begin{abstract}
:
According to the Census of India of 2001, India has 122 major languages and 1599 other languages are spoken by the 1.38 billion population of India. According to the Eighth Schedule of the Constitution, twenty-two (22) of them are considered as official languages; viz. Assamese, Bengali, Gujarati, Hindi, Kannada, Kashmiri, Konkani, Malayalam, Manipuri, Marathi, Nepali, Oriya, Punjabi, Sanskrit, Sindhi, Tamil, Telugu, Urdu, Bodo, Santhali, Maithili and Dogri. Translation services are essential for various sectors. As, most people only know one or two languages, translation services assist them in obtaining necessary information in their own tongue by eliminating the language barrier. In this study, we discussed the translation service in brief, its various types \& facets, working techniques and current status of translation services \& available centres in India.
\end{abstract}

Keywords: translation services, cover-to-cover translation, types of translation, selective translation, oral translation, Indian languages, translation and technology, machine translation, direct translation, transfer approach, interlingua approach, Indian Translation Research Centers, Library's role in Translation Services

\section{Introduction :}

Before discussing Translation Services we have to understand the meaning of the word "Translation".

The meaning of Translation is,

$\rightarrow \quad$ The process of translating words or text from one language into another.

$\rightarrow$ A written or spoken rendering of the meaning of a word or text in another language.

Today, literature is being published in various forms, subjects and languages. Literature, especially in the field of science and technology is published in so many languages. The scientists would also like to know what has been published in languages which is not known to them. They, therefore need a service to get such literature translated into a language known to them.

\section{Historical background (Demand arises) :}

Progress of science depends on the access to published science and technology(S\&T) literature. Published scientific literature serves as a foundation for further research in any area of scientific research. Access to this literature is, therefore, a fundamental right of researchers anywhere in the world. However, this is not true in practice. Over $50 \%$ of the scientific literature is published in languages other than English. The English speaking researchers do not have access to this literature unless it is translated in English. This problem was realised more acutely after the World War II, when government sponsored research got stimulus and great momentum. The seven major languages in which bulk of S\&T literature published were English, Russian, German, French, Japanese and Chinese. No country, however advanced, could afford to ignore scientific information produced in other countries. So there has been a great demand from researchers for translation of research results published in languages other than English. Many documentation Centers and special libraries attached to R\&D organisations started providing translation service to their scientists on demand. The demand for translation was particularly more for the journal articles. The major abstracting services cover S\&T literature published in languages other than English. These abstracting services provide abstracts 
in English of the articles published in other languages, so that scientists can judge the relevance of the article and get it translated if required. Similarly, for French and Russian speaking scientists, the major abstracting services in S\&T are 'PASCAL' and 'FRANCIS' from INISTCNRS, France in French language and 'Refratrivnyl Zhurnal' from VINITI, erstwhile Russia in Russian language.

When erstwhile USSR launched the world's first space satellite, the U.S. government looked for all sorts of explanations. One reason given for apparent Russian success was that Soviet scientists used the western literature, while western scientists did not use the Russian literature because they could not handle the Russian language. To solve this problem, the National Science Foundation undertook a large scale programme in support of 'cover-to-cover' translation of Russian publications. In India too NISCAIR (Formerly INSDOC) has been providing translation service from many foreign languages in English to the scientists since its inception in 1952.

Translation of the documents is carried out by the translators. For scientific translations, a translator should have a good knowledge of both the languages as well as of the subject to understand the terminology of the given subject. Earlier most of the translation work was done by the human translators. With the advent of computers, research in machine translation (MT) started by 1950s. Machine translation is the application of computers to the task of translating text from one natural language to another. During the last 70 years of research in machine translation has resulted in large number of MT systems for mainframe computer, personal computers and for the Internet.

\section{Meaning:}

Translation is conversion of one language into another language but retaining the original sense. The original language is known as the Source Language and the translation of document in another language i.e in Target Language.

\section{Definition:}

According to Rondom House Dictionary, "translation is to turn (something written or spoken) from one language into another."

\section{Need for Translation Service :}

Language is one of the barriers in the free flow of information. Due to language barriers, communication between two scientist of different countries of different languages becomes very difficult.

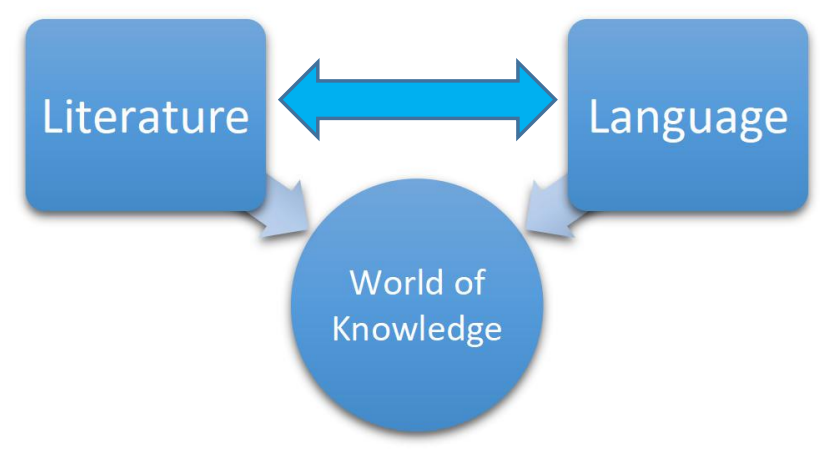

Figure 1: Interlinked Nature of Literature, Language \& World of Knowledge

As per an estimate by the National Lending Library of Great Britain, about half of world's literature is published in a language other than the English Language, especially in the field of science and technology. It is quite impossible for any scientists to study all language and read the published literature in their area of specialization. Persons like researchers, scientists, engineers and other 
scholars make use of translation service for their work. As they may not have the proficiency in more than one or two languages, there is need of translation services. Researchers also need translation of research papers which they came across in the course of literature search.

\section{Objectives of Translation Services:}

Some of the main objectives of translation services are as follow:

a) to provide a document in user language

b) to save the time of researchers, scientists and other scholars

c) to promote the use of documents

d) to promote cultural understanding

e) Establishment of cooperation and co-ordination with international organizations, agencies etc.

\section{Types of Translation Services :}

Translation may be of following types

- Cover to Cover (anticipatory service) translation $\rightarrow$ It is the translation of all the contents of the documents.

- Selective translation $\rightarrow$ The translation of the major points of the documents or selective partof the documents.

- Oral explanation/translation of the contents of the documents

\subsection{Cover-to-Cover Translation:}

A cover-to-cover translation is a complete translation of the document. It is generally confined to worth while journals. Such journals should be selected by National Translation Centre (NTS) in consultation with the subject specialist engaged in various $\mathrm{R} \& \mathrm{D}$ work in various institutions.

The main advantages of cover-to-cover translation is that it ensure the availability of important scientific literature irrespective of language barriers. It also eliminates the hit miss selection of articles.

One of the drawbacks of cover-to-cover translation is time lag. Time lag is the gap between publishing of a journal in its original language and its translated version. Some times, it ranges from six months to one year or even more.

\subsection{Selective Translation:}

A Selective Translation, as the name indicates, is a translation of selected parts of a document. This can include translations of tables of contents, lead paragraphs, abstracts, conclusions, or the sections of key interest.

\subsubsection{When is a Selective Translation appropriate?}

A selective translation is appropriate when:

i) We want to know the general content of a document, yet there are time constraints and/or we haveto operate under a limited budget;

ii) We are certain which parts of the document you want translated, and can indicate this clearly on the copy you provide to us. Only the indicated segments are then translated; 
iii) We know broadly what the document is about, but need to extract only certain information from it.

iv) We define what we want to know on the relevant subject matter, and we will identify and translate the appropriate sections.

\subsubsection{Advantages of a Selective Translation:}

a) Cost Effective: Selective translations represent a considerable cost saving over full translations and offer you maximum value for money, as you pay only for the information you actually need.

b) Time Saving: Selective Translations are produced much more quickly than full translations.

c) Translation Upgrades: Upgrade to a full translation later is available, if required.

Be Careful! The decision as to what sections to translate often relies heavily on your own, or the translator's judgment. There is a risk that important sections may be omitted. When requesting a selective translation, please indicate clearly what the relevant subject matter is, or which segments of the document you would like to be translated.

\subsection{Oral Translation:}

A Oral Translation is included in the services of almost every translation agency. Interpretation is required at conferences, meetings and in any event involving people that speak different languages. Interpretation is often put in the context of conference interpreting as it is most often used at conferences. It must be remembered that oral translation is provided by an interpreter and written translation by a translator.

\subsubsection{Types of interpretation (in oral translation)}

a) Consecutive interpreting - Speaker's text is interpreted usually by time slots of 5 to 15 minutes, depending on the difficulty of the text, interpreting by shorter phases is also called parroting. Consecutive interpretation requires no special equipment; interpreters use their notes taken during the speech.

b) Simultaneous interpreting - Interpretation is performed at interpreter's booth simultaneously with the speaker's presentation and the text in the target language reaches the listeners through special headphones. There are normally at least two interpreters in the interpreter's booth since one interpreter cannot interpret for more than half an hour on a continuous basis.

c) Whispered interpreting - Similar to simultaneous interpretation in that the interpretation takes place simultaneously with the speaking, but is characterised by no requirement of special equipment and the interpretation is whispered to only a few people.

d) Written text interpreting - In this case not spoken text is interpreted, instead, the source-language text is read from sheet and interpretation is given orally.

e) Sign language interpreting - As the name says the text is either interpreted into or from sign language in order to ensure the linguistic equality of all parties involved. In Estonia, this type of interpreting is provided by Estonian Association of Sign Language Interpreters.

\section{Differences between Written and Oral Translation:}

The first two types, i.e. Cover to Cover and Selective Translation are considered as Written translation. Although both written and oral translation deals with putting texts into a different language, written translation and interpretation differ in various aspects. The most evident differences among others are: 


\section{(i) Recorded or not}

Interpreter interprets once and nobody will hear, read or analyse it again. Written translation, however, is recorded, it can be repeatedly read, amended, analysed, retranslated, etc.

\section{(ii) Tools}

Interpreter's tools are mainly the source text and his or her own knowledge, while it is also important to guess what the speaker might say next. Translator, however, can use various dictionaries, other texts on the same subject and also expert advice and instructions. As the time for contemplation is very scarce in the case of simultaneous interpreting, interpreters may fall into difficulties when trying to convey e.g. jokes or idioms, as the detection of equivalents may require more time that is available in the interpretation process.

\section{(iii) Time for contemplation}

Simultaneous and whispered interpreting leaves minimum time to think, one must react immediately when the speaker has begun a sentence. Consecutive interpreting offers a bit more time, for interpreting is slightly delayed. Written translation provides as much time as stipulated by the deadline, and, as a rule, translator has time to go over the translation.

\section{(iv) Team work}

This only applies to simultaneous interpretation where each booth has two or three interpreters that help each other if necessary. Translators, however, mostly work alone, although they may consult with specialists, etc.

\section{(v) Client feedback}

In case of interpretation client is present and able to comment after interpretation on the merits and drawbacks of specific interpretation. In the case of translation the feedback does not occur immediately, and it may happen that client gives no feedback at all.

\section{Translation Process and Translator :}

Translation is the process of transferring the information contents of the text in one language (L1) into another language (L2). The former (L1) is called the 'Source Language (SL)' and the latter (L2) is called the 'Target Language (TL)'. In the translation process, the language expert analyses the given text in the source language from various view points including meaning, grammatical structure of the sentence(s), terminology, etc. and comprehends the information contents of the sentence(s). The more accurate the comprehension of the information contents of the sentence(s), the more precise will be its transfer in target language. If the translator fails to understand the message communicated by the source language, the information transferred into target language will either be distorted or inadequate. A translator, therefore, should have sufficient knowledge of the source language as well as of the target language. In addition, s/he should be a subject expert to understand the terminology of the given subject. Each discipline has its own peculiar jargons and terms and a good translator should have in depth knowledge of these terms in both the languages. Thus, subject knowledge is an essential requirement, specifically for translating S\&T documents.

\section{Translation Methods :}

The appropriate method of translating any text depends on the material to be translated. The Translation Methods are generally of two types, Literal Translation and Literary Translation. 
Material in science, technology and social sciences is translated adhering to the contents of the original. This is known as Literal Translation. Literal translation is utilised for factual type of materials such as commercial correspondence, legal materials, technical materials as well as scholarly materials in the pure and applied sciences and social sciences.

Whereas translation of materials in humanities such as novels, plays, poetry, films, television, radio, motion picture scripts and vocal music texts, etc. is Literary Translation.

Literal and Literary method of translation differs from each other in some ways -

a. The style and techniques of expressing feelings are more important in case of Literary Translation then Literal.

b. Second difference is the delivery of material in target language. Translation of literary material is destined for mass consumption. On the other hand scientific, technical, legal and commercial materials are intended for the specialists in a given field.

c. The other major difference is that science and technology translations are done once, while literary classics (such as novels, religious books like Bible, Bhagwat Gita, etc.) are repeatedly translated.

\section{Translation Services in Science and Technology (S\&T):}

Various disciplines of science and technology (S\&T) as well as newer interdisciplinary areas such as environmental studies, medical electronics, biotechnology, etc. are more in numbers than those in humanities and social sciences. Advances in these fields occur much more rapidly and must be reported to the researchers as soon as possible. Major share of research and development (R\&D) funding also goes to these knowledge areas. However, great portion of S\&T research is carried out in non-English speaking countries. Published literature is the most effective means of exchanging knowledge. Translations are the only solution in providing access to multi-lingual information resources.

Translation units formed an integral part of documentation and information centre of the organisation. Two kinds of services were organised within the information centre. They are :

i) In-house translation service to cater to the local translation requirements of the organisation from a few foreign languages into English; and

ii) General translation service which provided translation service to any individual or organisation from large number of foreign languages into English or vice versa on payment basis (e.g. Foreign Language Translation Service of NISCAIR)

\section{Translation Centers:}

In many countries national translation centers were set up to monitor translation activities in the country. These centers either carried out translation work themselves or acted as referral centers for collecting, processing and announcing the translations done by various agencies in the country. In this section you will study about the activities of some of the major translation centers which came up during 1950 s and 1960 s around the world.

In the United States, major translation efforts consisted of English language translation of S\&T material captured during the World War II, sponsored by National Science Foundation (NSF) and Special Library Association (SLA). During this period a large number of foreign reports were being translated simultaneously by different organisations which resulted in duplication of translation efforts, amounting to waste of time and money. The need was felt to establish a clearing house for translations, where translations could be collected, processed, announced and copies supplied on request. 
In response to this need, two translation centers viz. Scientific Translation Center and SLA Translation Center (Later named as National Translation Center) were set up where translations could be deposited.

1) Scientific Translation Center: NSF funded 'Scientific Translation Center' which covered Russian type scripts and technical reports that were deposited in Library of Congress. This Centre was located in Library of Congress.

2) SLA Translation Center(National Translation Center): Document Delivery Service National Translation Center was founded in 1953 under the name of SLA Translation Pool. This Centre engaged in collecting and processing translations from western European and Oriental languages. The Center was located in John Crerar Library, Chicago. Further it become a part of Library of Congress in 1989. The important publication of it was Translation Register Index since 1967. The Library of Congress had closed the National Translation Centre on September 30, the end of fiscal year 1993 .

3) International Translation Center (ITC): ITC came into existence in 1961 (Formerly known as European Translation Center) in Delft, The Netherlands. The present name was adopted in 1975. ICT centralized translation announcements made by participating agencies and published World Translation Index (WTI) - World Transindex since 1978. ICT published 10 issues of World Transindex per year. First issue of the WTI provided list of cover-to-cover translated journals.

4) Index Translationum (1932+): Published by UNESCO, Index Translationum is an international bibliography of translations. It provides details of books translated in the world into English covering all fields of knowledge. Each year, about 100 UNESCO Member Countries, send bibliographical details of books translated in their country to the UNESCO Secretariat. UNESCO brings out Index in print annually. From 1979 onwards, UNESCO is maintaining the machine readable database of this Index. The database contains cumulative bibliographical details of over 2,000,000 books translated and published in about 100 member states of UNESCO.

\section{Translation Centers and Translation Service in India:}

In India several Governments and Public Sector Organisations, R\&D institutions in S\&T have in-house translation facility to meet their own translation requirements for limited number of languages. Some such organisations are BARC, DESIDOC, ONGC, BHEL, MECON, HAL etc.

1) Institution of Asian Studies: It involved in research, training, translation and publication activities in Asian languages. The institute carries out study and research in Tamil, Kannada, Japanese, Telugu and Buddhism and related translation and publication activities. In addition, the Institute is carrying out research in Manuscriptology and Folklore studies and translation activities related to these areas. It is located in Chennai.

- Available at: http://www.instituteofasianstudies.com

2) Indian Council for Cultural Relation (ICCR): It Carries out literary translation of books and other documents to project Indian cultural heritage to the world. The Council's translation and publication activities focus on books related to Indian culture, philosophy, mythology as well as music, dance and theater. The council translated Sanskrit classics into a number of foreign language including French, Spanish, Arabic, Russian and English.

- Available at: http://www.iccrindia.net

3) Central Institute of English and Foreign Languages: It conduct training courses in foreign languages including French, Spanish, Arabic, Russian and English. 
4) NISCAIR Foreign Language Translation Service: NISCAIR (formerly INSDOC) has been providing translation service to S\&T community since its inception in 1952. It caters to the translation requirements of National Laboratories, S\&T Institutes, R\&D Organisations, Corporate and Public Sector Undertakings, Universities, Research Scholars, etc. NISCAIR provides translations of S\&T documents from 20 foreign languages into English. The languages include Chinese, Czech, Danish, Dutch, French, German, Hungarian, Italian, Japanese, Norwegian, Polish, Portuguese, Rumanian, Russian, Serbo-Croatian, Spanish, Swedish, etc. NISCAIR provides reverse translation (from English into foreign languages) also. Translation of English text (maximum of one page), into French, Russian and Spanish is undertaken on request. Translation of full English document into Japanese is provided on request. NISCAIR also provides interpretation services in Japanese language. Charges for translation services for different languages are available at NISCAIR website.

- Available at: www.niscair.res.in/activitiesandservices/Services/services.htm

5) Indian Statistical Institution, Kolkata: ISI enables successful communication through full-service language and localization solutions, from a global team of linguists deeply rooted in the cultural and technical nuances of virtually every language used in business. Founded in 1982, ISI combines the latest technology with expert human touch at every stage to enable successful communication in more than 100 languages(119). ISI serves a wide range of industries and organizations, specializing in health-care, life sciences, pharmaceutical and financial services. ISI Translation Services is an industry leader specializing in culturally and linguistically appropriate translations for the health care market. ISI was one of the first to address the special linguistic and cultural needs of both non- and limited-English-proficient communities of the United States.

- Available at: http://isitranslation.com/

6) Defense Scientific Information \& Documentation Centre: DESIDOC provides translation service to DRDO Scientists and at present can handle translation jobs from 14 foreign languages in to English. The centre regularly scans selected foreign language periodicals, identifies articles of defence interest and translate abstracts of these articles in English. It brings out a quarterly publication titled, 'Science Abstracts from Foreign Language Journals', and provides full translation of articles covered in this publication, as demanded.

- Available at: https://drdo.gov.in/drdo/pub/techfocus/aug98/transl.htm

Some other institutions like; IISC, Bangluru BARC, Mumbai... etc.

\section{Translation Activities in Humanities and Social Sciences in India:}

No one fully understands the meaning of 'Unity in Diversity' better than people in India. There are twenty-four official languages recognised by Government of India. In addition to that, there are about 2000 dialects that have been recognised in India. Quite a lot of communication is done in English and most state governments function in their own regional languages. This situation demands an urgent need for translation of official documents. Apart from official documents, there is need for translation of text books, scholarly materials, literary materials, etc. In response to this need, a number of organisations in India are actively involved in translation activities in the fields of humanities and social sciences.

Some examples given below: 
a. The National Council of Educational Research and Training (NCERT) and National Book Trust (NBT) (http://www.ncert.nic.in/)

b. State Council of Educational Research and Training (SCERT) (http://www.edudel.nic.in/scert.html/)

c. Sahitya Akademi, est.1954 (http://www/sahitya-akademi.gov.in/)

d. Central Institute of English and Foreign Languages (CIEFL), est. 1958 (http://www.hyderabadeducation.net/university) ...etc.

\section{Machine Translation:}

Machine Translation (MT) is application of computers to the task of translating text from one language to another.

In MT system, the computer program analyses the text in one language - the "source language" and then produces the equivalent text in another language - the "target language", without human intervention. Machine translation is also referred to as "automatic translation". The systems for automatic translations have been under development for over 60 years. The first public demonstration of MT system was held in New York at the head office of IBM in 1954. This MT system translated 49 selected Russian sentences into English in the field of chemistry. This demonstration stimulated the funding of MT research not only in the U.S. but also worldwide.

Currently, the state of machine translation is such that it involves some human intervention at pre-editing or post-editing phase. This means the translation produced by MT systems must be revised or "post-edited" by human translators to achieve publishable quality. Sometimes, such revision may be substantial as MT system produces only 'draft' translation. In other words, machine translation is not perfect and as results will never be able to compete with human translator. However, in the fields with limited range of vocabulary and simple sentences, MT systems are producing good results.

For instance, TAUM, a Canadian MT system, translates weather reports from English to French without any human intervention.

Earlier the MT systems were based on "direct translation" via bilingual dictionaries with very little analysis of syntactical structure of a language. By 1980s, advances in computational linguistics offered better facilities for machine Translation Service translation. Rule-based method could be used to carry out machine translation. In these systems, the text of the source language is analysed into abstract representation of 'meaning' of the text involving number of programmes to identify word structure (morphology), sentence structure (syntax), recognise correct semantic relationship and distinguish between homonyms, phrases and other ambiguities. Syntactical analysis of a language involves identifying use or function of a word, phrase or clause in a sentence.

For example, English word such as 'light', which can be a noun, adjective or verb; or 'solution' which can be a mathematical term or a chemical term; or 'plant' which can be a botanical term or industrial term.

\section{Types of Machine Translation:}

At present, most of the MT systems can be grouped into 3 basic system type: Direct, Transfer, and Interlingua.

\subsection{Direct Translation:}


Direct translation is perhaps the most common pitfall among translators and often happens when a translator is fatigued or in a rush. For example, when the source language is mirrored, sometimes word for word, into the target language. If a text is translated like this, although each word may be translated correctly, the overall meaning of the original text is, quite literally, "lost in translation".

\section{Example:}

The MT systems with this type of translation using only bilingual dictionaries. The best known MT systems for mainframe computers are of 'direct translation type' e.g. SYSTRAN, LOGOS, FUJITSU ATLAS Systems. They are, however, improved version of this type. They are highly modular in construction and easily modifiable and extendable.

SYSTRAN system, originally designed for Russian to English, is now available for 52 language combinations.

LOGOS originally marketed for German to English is also now available for other languages, such as English to German, Italian and Spanish and from German to French and Italian. FUJITSU ATLAS System translates from English to Japanese and from Japanese to English.

\subsection{Transfer Approach:}

In this approach there are three basic stages:

i) analysis of input text into abstract source representation,

ii) transfer to the abstract target representation, and

iii) generation into output language.

In this approach three dictionaries are needed: i) a source language dictionary (SD), ii) a target language dictionary (TD), and iii) a transfer dictionary i.e. bilingual dictionary (STD). Source language dictionary (SD) analyses the source language text, sentence by sentence. Bilingual dictionary transfers the analysed source text into target language. Then target dictionary generates the text into target language. In this type of system, transfer stage requires bilingual dictionary for each set of language pair. In a multilingual environment, the number of transfer blocks required would be equal to the number of languages a MT system covers.

Example: METAL from Siemen Company from Germany (Now with GML and LANT Company) is a commercial MT system based on the 'transfer' approach.

\subsection{Interlingua Approach:}

In this system the source text is analysed into abstract representation, which is designed to be a kind of language independent 'Interlingua' and can serve as an intermediary between large numbers of natural languages.

Here the translation is done in two stages:

i) from source language to Interlingua and

ii) from Interlingua to the target language.

Example: The example of Interlingua system is MT system by Cordier and Mograbhi which translates cooking recipes from French into Arabic.

Apart from the above mentioned techniques of the MT systems, machine translation can be done by using other techniques such as rule-based translation, example-based translation and statistical machine translation ... etc. 
1) Rule-based Translation: The most common technique to use MT is by coding grammatical rules of source and target languages in the software and get the translation done using these rules and dictionaries specifically created for this purpose.

2) Example-based Translation: The other technique to use machine translation is by storing the source and target language pairs as example base and then match the new sentences for similarities from the example base. Translation obtained from the best match is called Example-based Translation Method.

3) Statistical Machine Translation: In addition, statistical methods can be employed to increase the efficiency of the translation. Statistical Machine Translation is a relatively new technique. It is not yet widely use. It uses collections of documents and their translations to Translation Service 'train' software. Over time, these data driven systems 'learn' what makes a good translation and what doesn't and then use probability and statistics to decide which of possible translations of a given word or phrase is most likely correct based on the context.

4) Tree Adjoining Grammar based MT: The Tree Adjoining Grammar (TAG) consists of a set of elementary trees, divided into initial and auxiliary trees. This works on tree-to-tree translation model and makes use of syntactic tree for both the source and target language. Parser and Generator modules recognize various grammatical entities in the English sentence, analyze them, represent them in different tree structures and synthesize equivalent Indian Language sentences on the basis of the derivational tree structure and the Transfer Grammar.

5) Analyze-Transfer-Generate based MT: The source languages text is preprocessed (collected, cleaned, and formatted) and analyzed. After language analysis, transfer of vocabulary and analyzed structure is carried out. And finally the target language text is generated.

6) Hybrid \& Pseudo Interlingua MT: This technology uses a pseudo Interlingua approach. It analyzes English only once and creates an intermediate structure with most of the disambiguation performed. The intermediate structure is then converted to each target language through a process of text generation. It also uses rule based MT and Example based MT approaches.

\section{MT Systems for Mainframe, Personal Computers (PC) and the Internet:}

Most widely known commercial MT Systems like SYSTRAN, METAL, LOGOS and FUJITSU ATLAS initially developed for mainframe computers, have also brought out their PC based versions. SYSTRAN Company offers a wide range of PC products such as SYSTRAN Professional, SYSRAN Personal, SYSTRAN Office Translator and SYSTRAN Web Translator. The SYSTRAN MT systems with large dictionary databases and large number of languages have advantages over other PC based systems.

At present, there are four types of translation demands from MT systems: (i) Use of MT for Dissemination; (ii) Use of MT for Assimilation; (iii) Use of MT for Interchange; and (iv) Use of MT for Information Access Systems.

i) MT Systems for Dissemination: This type of demand is made to have quality translation for publication purposes. To fulfil this demand, most of the MT systems produce translation which must be revised or 'post-edited' by human translators to achieve publishable quality. In recent years, the MT systems for dissemination purposes have been augmented by developments in translation tools (e.g. terminology databases, translation memories, etc).

ii) MT Systems for Assimilation: As mentioned earlier, MT systems produce draft translation and it must be revised by human translator to reach publishable quality. However, at times, users are satisfied with draft translation produced by MT systems because they can extract or assimilate 
what they need to know from the unedited version of the translation. With the coming of cheaper PC-based MT systems in the market, this type of use has substantially increased.

iii) MT Systems for Interchange: This demand is for translation of electronic text on the Internet, such as translation of web pages, electronic-mail and electronic 'chat'. This type of demand is increasing rapidly. To fulfil this demand there is need for immediate translation to convey the basic contents of the message. Here, MT systems are playing major role, since they can operate virtually. These systems carry out translation in-real time and online. People are using MT systems for this purpose and have no objection to the poor quality of translation, because it fulfills their immediate need. Another area of MT research is the development of systems for spoken language translation e.g. in telephonic conversation and in business negotiations.

iv) MT Systems for Information Access Systems: This demand is for integration of translation software into:

a. Systems for the search and retrieval of full-texts of documents from databases (e.g. retrieval of translated electronic version of journal articles in science, medicine and technology or for retrieval of bibliographic information);

b. Systems for extracting information (e.g. product details) from texts in particular from newspaper reports;

c. Systems for summarising texts; and

d. Systems for integrating non-textual databases. This field is the focus of a number of research projects in Europe. The aim is to provide access, to all members of European Union,to the sources of data and information, whatever may be the source language.

\section{Machine Translation Research in India:}

India is a linguistically rich country with 24 official languages, each of which is spoken by millions of people in the country. Since most of the information is generated in English and Hindi constitute bulk of correspondence in government offices, these languages have been identified as priority area

of research in machine aided translation in India. Accordingly, two specific areas of research have been identified :

i) MT systems for translation between Indian languages, and

ii) MT systems for translation between English to Hindi.

Realising the immense potential of MT, Department of Official Language (DOL), Government of India, began actively funding projects in MT research.Government of India has facilitate development of Machine Translation Systems for translation from English to Indian Languages and from Indian Languages to Indian Languages using different technological approaches.

1) The language pairs addressed for English to IL are English-Hindi; Assamese; Bodo; Bengali; Gujarati; Malayalam; Marathi; Nepali; Punjabi; Oriya; Tamil; Telugu; and Urdu.

2) Indian Language MT Systems include 9 bidirectional language pairs,

\begin{tabular}{|c|l|c|l|}
\hline SN & Name of Pair & SN & Name of Pair \\
\hline 1 & Punjabi - Hindi -Punjabi & 5 & Marathi - Hindi - Marathi \\
\hline 2 & Telugu - Tamil - Telugu & 6 & Bengali - Hindi - Bengali \\
\hline
\end{tabular}




\begin{tabular}{|l|l|c|l|}
\hline 3 & Urdu - Hindi - Urdu & 7 & Tamil - Hindi - Tamil \\
\hline 4 & Hindi - Telugu - Hindi & 8 & Kannada - Hindi - Kannada \\
\hline 9 & Malayalam - Tamil - Malayalam \\
\hline
\end{tabular}

Table 1: Nine (9) Bidirectional Language Pairs of Indian MT System

Currently, institutions in the country namely C-DAC, Mumbai and Bengarulu and IITs have taken the lead in developing MT systems. Ministry of Information Technology has identified the following domains for the development of domain specific translation systems :

i) Government administrative procedures and formats;

ii) Parliamentary questions and answers;

iii) Pharmaceutical information; and

iv) Legal terminology and judgments

Some brief descriptions of Indian MT research initiatives are discussed here;

1) C-DAC (Centre for Development of Advanced Computing) Mumbai and Bengaluru (erstwhile National Centre for Software Technology (NCST) Mumbai) is a scientific society under the aegis of Department of Electronics and Information Technology undertaken projects in the area of machine translation is called E-ILMT (English to Indian Languages: Translation Service Machine Translation System). The main objective of the project is to remove language barriers in a country like India where there are 24 official language. (http://www.cdacmumbai.in/index.php/cdacmumbai/)

2) Department of Computer Science and Engineering of IIT, Kanpur has developed two machine translation systems for translating English text in Indian languages. These MT systems are ANGLABHARTI and ANUBHARTI. ANGLABHARTI system is based on pattern directed approach. ANUBHARTI system of MT is based on example-based approach.(http://www.iitk.ac.in)

3) 'Anusaarka' is a machine translation system developed by Chinmaya International Foundation, IIT Hyderabad and University of Hyderabad, Department of Sanskrit Studies. Anusaarka is a Sanskrit word which means to follow. It is an English Hindi language accessing software. This helps the users to have access to the translated text in any Indian language from the source language(s) i.e. English or any Indian regional language.(http://anusaarka.iiit.ac.in)

4) The Technology Development for Indian Languages (TDIL) Programme was launched by Ministry of Information Technology, Govt. of India in the year 1991-1992. The Programme aims at promotion and development of computerbased translation tools for Indian languages.Currently,Ministry of Information Technology is sponsoring about $75 \%$ of the MT projects in India. (http://www.tdil.mit.gov.in/)

The slow development of MT systems in India is due to lack of lexical resources for Indian languages. Indian groups are now addressing this challenge jointly by starting a collaborative open source initiative called 'LERIL (Lexical Resources for Indian Languages), which includes several groups such as IIIT Hyderabad,NCST (now C-DAC Mumbai), Mumbai, Kendriya Hindi Sansthan, etc. Sharing of resources would help MT projects to take off at a faster rate.

\section{Library's Role in Facilitating Translations:}

Libraries can play an active role in meeting user's demands for translation. They should have information about translation pools, translation centers, professional associations, Government agencies, commercial publishers and their products (including cover-to-cover translated journals.), directories of translators and translating firms. The library should have these sources: both published 
as well as online, on up-to-date basis and it should be able to assist the users in identifying institutions holding the needed translations. If demands are very frequent, then library staff members or resource persons who are available when needed, should be able to either translate or abstract or provide summaries of the material needed to meet urgent need.

\section{Summary:}

Translation service is one of the most responsive services of libraries and information centers. The spectacular growth in information technology, the widening reach of the Internet, the expansion of trade globally and ever increasing scientific and cultural cooperation have undoubtedly increased the demand for translation service. However, reduction in funding for information services has resulted in significant decline in translations made on ad hoc bases. This perhaps may be one of the reasons which have led to the closure of International Translation Center as well as its prestigious publication World Translation Index.

Research in machine translation has resulted in number of MT systems for mainframe, personal computers and the Internet. The development of computerbased translation tools such as multilingual dictionaries, terminological databanks, multilingual word processing systems and translation memories are greatly facilitating the translation process for both human translators as well as for automatic translation. Demand for translation of electronic text on the Internet such as translation of web pages, electronic mail, and electronic 'chat' is rapidly increasing. Here, MT systems are playing major role by providing translation on line and in-real time. In India, research in machine translation is in progress in two areas viz., MT systems for translation between Indian languages and MT systems for translation between English to Hindi. Currently, the institutions in the India namely C-DAC and Indian Institute of Technology are actively involved in MT research.

\section{References :}

1. NOPR:Home, nopr.niscair.res.in/bitstream/123456789/28085/1/ALIS\%2026\%281-4\%29\%2052-56.pdf.

2. Indian Language Technology Proliferation and Deployment Centre Home,tdil-dc.in/undertaking/article/262568Machine\%20Translation\%20Acceptance\%20Ver4.0. pdf.

3. "Direct Translation -." Translation Agency with Satisfaction Guarantee on Translations, www.copypanthers.com/resources/direct-translation/. Accessed 28 Feb. 2018.

4. Krishan Kumar. Reference Service. 5th rev.ed. Delhi : Vikas, 2007

5. Kumar, P.S.G. Fundamental of information science. New Delhi : S Chand, 1998 